\title{
Modelo de partería, justicia reproductiva y atención de aborto en México
}

\author{
Midwifery Model of Care, Reproductive \\ Justice, and Abortion Care in Mexico
}

Fecha de recepción: 06/09/2021

Fecha de aceptación: 26/11/2021

Fecha de publicación: 07/03/2022

https://doi.org/10.48102/if.2022.v2.n1.198

\author{
Hannah Borboleta* \\ hannah.borboleta@moradavioleta.org \\ ORCID: https://orcid.org/0000-0002-1545-334X \\ Partera y directora clínica de Morada Violeta \\ Licenciada en Sociología y Antropología \\ Morada Violeta \\ México
}

Kay Nicté Cisneros García**

kay.cisneros@moradavioleta.org

ORCID: https://orcid.org/0000-0003-3674-0441

Partera en Morada Violeta

Morada Violeta

México

* Hannah Borboleta es licenciada en Sociología y Antropología por la Universidad de Lyon (Francia); se formó como partera con mentoras en Xalapa y en Luna Maya CDMX (México) bajo los estándares y competencias del North American Registry of Midwives (NARM). Ha escrito sobre partería en su blog Partera en bici (www.parteraenbici.com) y en medios como MUNet (https://www.midwiferyunitnetwork.org), Luchadoras (https://luchadoras.mx) y GIRE (https:// gire.org.mx). Sus publicaciones más recientes son "Emergent change in a Mexican midwifery center organization amidst the COVID-19 crisis" (Alonso et al., 2021), "Our midwife friends in Mexico City" (Borboleta, 2021), "Atravesar las crisis, enseñanzas de la partería” (Borboleta, 2020), "El parto puede ser acompañado. ¿Y luego?" (Borboleta, 2019). Es experta en partería feminista, salud sexual y reproductiva, aborto desde el modelo de partería y mentoría de parteras.

** Kay Cisneros es partera feminista en Morada Violeta; realizó su formación como partera en Luna Maya CDMX bajo los estándares y competencias del NARM. Su práctica se enfoca

Borboleta, H., Cisneros, K. y Llanes, R. (2022). Modelo de partería, justicia reproductiva y atención de aborto en México. Iberoforum, Revista de Ciencias Sociales, Nueva Época, 2(1), 1-17, Ensayos, e000198. https://doi.org/10.48102/if.2022.v2.n1.198 


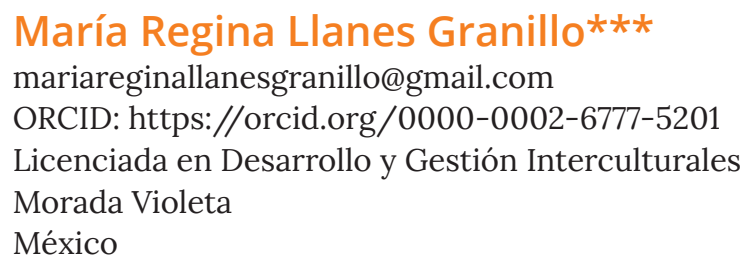

\section{Resumen}

En este texto, parteras mexicanas que acompañamos la salud sexual y reproductiva de las mujeres exponemos los beneficios del modelo de partería y de la atención por parteras en los servicios de aborto. Explicamos cómo este modelo fomenta el respeto y la defensa de los derechos humanos y proponemos que es una herramienta para garantizar el ejercicio de justicia reproductiva de las mujeres, así como una solución contra la violencia ginecobstétrica que ejerce el personal médico en los servicios de interrupción del embarazo. Describimos cómo las parteras han acompañado históricamente procesos de aborto y analizamos cómo, en la actualidad, el que parteras realicen abortos es una competencia promovida por diferentes organismos internacionales y ya ocurre en algunos países. Exponemos experiencias de parteras que proveen servicios de interrupción y acompañamiento a las mujeres en estos procesos y argumentamos por qué también es una necesidad en México.

\section{Palabras clave}

Modelo de partería, aborto, justicia reproductiva, violencia obstétrica, parteras.

\section{Abstract}

In this paper, Mexican midwives who accompany women's sexual and reproductive health explain the benefits of the midwifery model and midwifery

en la atención en casa a la salud sexual y reproductiva de las mujeres dentro del modelo de partería. Su publicación más reciente es "La partería: una práctica para el futuro" (Cisneros, 2019).

*** Regina Llanes es licenciada en Desarrollo y Gestión Interculturales por la Universidad Nacional Autónoma de México (UNAM); realizó su tesis profesional sobre violencia obstétrica en el modelo hospitalario de atención al nacimiento. Ha participado en organizaciones de la sociedad civil dedicadas a la defensa de los derechos sexuales, reproductivos y no reproductivos de las mujeres. Es aprendiz de partera en Morada Violeta; está en el último nivel de la formación como partera primaria en supervisión clínica. 
care in abortion services. We explain how this model promotes respect and defense of human rights and propose that it is a tool to guarantee the exercise of reproductive justice for women, as well as a solution against obstetric violence exercised by medical personnel in pregnancy termination services. We describe how midwives have historically accompanied abortion processes and analyze how, nowadays, midwives performing abortions is a competence promoted by different international organizations and already takes place in some countries. We present experiences of midwives who provide abortion services and accompany women in these processes and argue why it is also a necessity in Mexico.

\section{Keywords}

Midwifery model of care, abortion, reproductive justice, obstetric violence, midwives.

\section{Introducción}

Las mujeres han abortado desde el principio de los tiempos; las restricciones que han surgido en los últimos siglos sobre el aborto, lejos de desaparecerlo, sólo lo han vuelto clandestino y, para muchas mujeres, más peligroso. A la par de las prohibiciones gubernamentales, han surgido redes de mujeres que acompañan a otras en sus procesos de abortos; entre esas acompañantes están y siempre han estado las parteras.

En el mundo, alrededor de 56 millones de mujeres abortan cada año (Singh et al., 2018) y no hay suficientes profesionales de la salud para acompañarlas. Desde hace décadas, instancias como la Organización Mundial de la Salud (OMS), colectivos en el mundo entero y diversos artículos académicos constatan que hay una escasez de proveedoras de aborto (World Health Organization [WHO], 2015). Como consecuencia de la pandemia por COVID-19, la falta de personal se ha recrudecido, pese a que la Secretaría de Salud de México declaró el aborto como servicio esencial. Además, la violencia ginecobstétrica suele estar presente en los servicios de aborto y permea la calidad de atención que reciben las mujeres: "Me metió los dedos para ver cuántas semanas tenía y me dolía y le dije que parara y no paraba", dice una usuaria de servicios de aborto legal en la Ciudad de México (Colega partera, Comunicación personal, 13 de julio de 2021). En este contexto, muchas mujeres terminan abortando solas, con miedo y con procedimientos que pueden poner en peligro su salud. 
En la Ciudad de México, de 2007 a 2021, 240916 mujeres accedieron a un aborto legal (Secretaría de Salud, 2021) y no hubo ni una sola muerte. La mayoría de los abortos, al ser procesos de bajo riesgo, pueden abordarse desde el primer nivel de atención y ser atendidos por parteras. Invertir en el reconocimiento y la capacitación de las parteras para que provean servicios de aborto y acompañen a las mujeres sería benéfico y costo-efectivo, aumentaría las opciones disponibles y la seguridad, reduciría la violencia que las mujeres reciben en la atención, contribuiría a disminuir la saturación del sistema hospitalario y mejoraría la calidad de la atención en los servicios de aborto.

En el presente artículo, parteras mexicanas que damos atención a la salud sexual y reproductiva, argumentaremos que el reconocimiento de las parteras como personal capacitado para atender abortos es una necesidad en México. Tomamos como marco teórico la justicia reproductiva para describir el modelo de partería (MdP), exponer la evidencia y las recomendaciones de diversos organismos alrededor del mundo sobre sus beneficios y por qué es importante contar con él en la oferta de servicios de aborto.

\section{Contexto histórico: la atención al aborto por parteras}

Por milenios, la interrupción del embarazo fue un suceso que ocurría dentro del ámbito de lo privado; aunado a ello, la falta de conocimiento respecto al inicio de la vida embrionaria hacía que los brebajes y otros métodos utilizados como anticonceptivo o para realizar un aborto no tuvieran una distinción clara (Núñez, 2018).

Ha sido ampliamente documentado que las parteras, "matronas", "curanderas" o "mujeres sabias" fueran las creadoras y transmisoras del conocimiento femenino en torno al control de la reproducción, así como las encargadas de acompañar a las mujeres no sólo en la atención del embarazo, parto y postparto, sino en procesos de aborto y de decisión respecto a la reproducción (Federici, 2004).

El desplazamiento de las parteras en la atención a la salud de las mujeres fue un proceso que se recrudeció a partir de la necesidad del Estado burgués de controlar el crecimiento y reproducción de la población con la finalidad de asegurar la fuerza de trabajo necesaria para el crecimiento económico de la nación (Federici, 2004). Como consecuencia, desde el siglo XVI, en diferentes Estados europeos, comenzaron a culpar a las mu- 
jeres por ejercer control sobre su reproducción y sexualidad; así, se conceptualizaron como crímenes reproductivos, principalmente, el aborto, el infanticidio y la anticoncepción, los cuales eran penados con la muerte (Federici, 2004).

Dicho proceso se llevó a cabo a través de la creación de la figura de la bruja. Éstas eran, por un lado, las parteras: mujeres con conocimientos sobre la reproducción de las mujeres, acusadas de realizar abortos y cometer infanticidio como ofrenda al diablo (Federici, 2004). También eran brujas las mujeres que no deseaban reproducirse o que ejercían su sexualidad con fines no procreativos (Federici, 2004).

La estigmatización, la acusación y el asesinato de las mujeres que escapaban de la normativa patriarcal de la época se dieron mediante una caza de brujas, en la cual miles de mujeres, entre ellas un gran número de parteras, fueron quemadas en la hoguera (Federici, 2004).

En la Nueva España, no se empleaba coloquialmente la palabra aborto; se hablaba de "malos partos", lo que hacía referencia a los embarazos que no llegaban a término y a que las "criaturas" no habían sido bautizadas (Enciso, 2010). Eran atendidos por parteras y, en algunos casos, por médicos (Enciso, 2010). A nivel legal, se aplicaron códigos normativos europeos, realizados tanto por juristas de la Corona española como por eclesiásticos, todos ellos hombres, dada la nula libertad histórica de las mujeres de participar en estas esferas de toma de decisión. Dichos códigos sancionaron el aborto y lo conceptualizaron como un pecado en caso de que se hiciera de manera voluntaria (Núñez, 2018).

Entre los siglos XVI y XVIII, en medio de la estigmatización y el desplazamiento del ejercicio de las parteras, se consolidó la ginecobstetricia como especialidad médica; los médicos -únicamente hombres en un inicio- empezaron a atender tanto partos como la salud de las mujeres (Federici, 2004). Además, se institucionalizó la atención de la salud de las mujeres, que pasó de los hogares a los hospitales para que el Gobierno pudiera controlar la vida de las ciudadanas desde su inicio (Stern, 2002). Este desplazamiento se logró mediante una campaña de estigmatización respecto a la competencia de las parteras, lo cual funcionó para ocultar los motivos estatales subyacentes de control poblacional (Stern, 2002). Dicha campaña de estigmatización se vio reforzada con el hecho de que las parteras, históricamente, realizaran y acompañaran abortos. 
Como resultado de este proceso de desplazamiento y marginación de las parteras, los grupos médicos masculinos expropiaron y anularon los conocimientos y saberes que, por milenios, las mujeres habían creado alrededor de su cuerpo y su autonomía reproductiva. Sin embargo, desde el siglo XVI y hasta el XXI, las parteras continuaron realizando abortos, principalmente para mujeres pobres, campesinas, obreras y de la creciente clase media (Federici, 2004).

En México, durante el siglo XX, el Estado continuó con el desplazamiento de las parteras, motivado por pensamientos eugenistas para controlar a la población a través de los médicos y para que el cuidado femenino de la salud fuera sustituido por las enfermeras (Stern, 2002).

\section{Violencia ginecobstétrica \\ en la atención del aborto en México}

Arguedas (2014) define la violencia obstétrica como "un mecanismo de control y opresión, derivado del ejercicio del poder obstétrico - una forma de poder disciplinario- que produce cuerpos sexuados y dóciles" (p. 147), a través de "un conjunto de prácticas que degradan, intimidan y oprimen a las mujeres y a las niñas en el ámbito de la atención en salud reproductiva y, de manera mucho más intensa, en el periodo del embarazo, parto y postparto" (pp. 146-147).

Las cifras sobre violencia ginecobstétrica en México son limitadas. No se tienen registros oficiales y contundentes sobre violencia ginecobstétrica en otros ámbitos fuera de la atención del parto. La ausencia de estos datos nos impide hacer un análisis estadístico sobre la violencia ejercida hacia las mujeres en la atención del aborto en México.

Sabemos que, en los lugares donde el aborto está tipificado como delito, las mujeres que acuden a los servicios de salud por un aborto espontáneo o por complicaciones en un aborto inducido (generalmente relacionadas al acceso limitado a procedimientos seguros) se exponen a diversas manifestaciones de violencia ginecobstétrica; suelen ser cuestionadas y maltratadas, se les niega la atención y, en algunas ocasiones, son referidas a las autoridades para ser investigadas. De 2007 a 2016, se dictaron noventa y ocho sentencias a mujeres por el delito de aborto; en muchos casos en los que hubo sentencias, el aviso a las autoridades fue dado por los profesionales de la salud a pesar de que las mujeres referían estar presentando 
un aborto espontáneo o no tener conocimiento del embarazo (Grupo de Información en Reproducción Elegida [GIRE], 2018).

En México, el aborto hasta las doce semanas está despenalizado en la Ciudad de México, Oaxaca, Veracruz e Hidalgo; sin embargo, su legalidad no garantiza completamente el acceso a una atención digna y de calidad. En nuestra práctica, constantemente escuchamos relatos de mujeres víctimas de violencia ginecobstétrica en la atención a sus abortos, la cual se expresa como juicios sobre sus prácticas sexuales, desacreditación de sus emociones o sensaciones, manipulación emocional para conservar el embarazo, culpabilización, humillación, falta de sensibilidad, agresiones verbales, poca información sobre procesos y procedimientos, intervenciones innecesarias - como tactos vaginales excesivos o procedimientos como la dilatación y curetaje (legrado) ${ }^{1}$ en circunstancias que no ameritan ese nivel de intervención- y anticoncepción o esterilización forzada después de los procedimientos. Esto no sólo es nuestra experiencia, sino que ha sido documentado por GIRE y otras organizaciones, aunque, como ya señalamos, falta mucha sistematización del tema.

Todo esto tiene un impacto negativo en la salud física y emocional de las mujeres, a corto y largo plazo, y contribuye a que se expongan a acompañamientos inseguros para la terminación de un embarazo no deseado o a que no reciban atención en una situación de aborto espontáneo, lo cual puede poner en riesgo sus vidas.

La violencia ejercida por parte del personal y las instituciones de salud no puede analizarse como casos aislados: es un problema estructural e institucionalizado con orígenes diversos y complejos. Desde diferentes disciplinas como la antropología y la sociología médica, se ha analizado cómo el campo médico deviene de y se rige, hasta la fecha, por estructuras patriarcales, jerárquicas y autoritarias; ha tenido un papel histórico como estructura de control, vigilancia y socialización (Ehrenreich y English, 1990). La relación médico/a-paciente es una interacción de poder desigual mediada por diversos elementos: por un lado, la diferenciación entre la figura del médico/a como poseedor del conocimiento ("experto") en contraste con

1 La OMS recomienda la aspiración por vacío y el aborto con medicamentos para la atención del aborto antes de las doce semanas, y menciona que el legrado (ampliamente realizado en México) es una práctica obsoleta y no recomendada de manera rutinaria (Organización Mundial de la Salud [OMS], 2020). 
la figura de la/el paciente como un agente pasivo y "no experto"; por otro lado, las diferentes subjetividades que atraviesan a cada persona, como el género, la clase, la raza, la edad, entre otras (Castro y Erviti, 2015).

En lo que respecta a la atención a la salud sexual y reproductiva, la institución médica, en tanto expertos con una fuerte autoridad moral en las sociedades, ha contribuido a reafirmar los estereotipos y roles sociales de género en torno al papel reproductivo y de crianza de las mujeres, aportando a la construcción y reproducción de los mandatos sociales referentes a la maternidad y la sexualidad femenina, así como sancionando las prácticas que no cumplen con dichos mandatos (Palomar, 2004).

El carácter autoritario de la práctica médica que deriva en la pérdida de autonomía y agencia por parte de las usuarias, las estructuras basadas en estereotipos de género que permean la formación y el ejercicio médico, el entrenamiento para una atención distante que impide la conexión emocional con las usuarias (Castro y Erviti, 2015) dan paso a un enfoque de atención que se centra en la enfermedad y que contempla únicamente la dimensión física de la salud sin incluir su dimensión emocional. La saturación de los servicios hospitalarios, el estado de burnout del personal de salud y un Estado que no tiene como prioridad defender los derechos de las mujeres son el caldo de cultivo perfecto para el ejercicio de la violencia de género y la violación de los derechos humanos en la atención a la salud, en especial en las prácticas que desafían las normativas y los roles de género como el aborto.

\section{Justicia reproductiva y acceso a servicios de aborto}

La justicia reproductiva busca que los servicios de salud, incluido el acceso al aborto, sean accesibles, entendibles, dignos y seguros para cualquier mujer (Ross y Solinger, 2017, p. 9). Para lograrlo, es necesario que haya personal de salud capacitado sobre la atención a la salud desde el marco de derechos humanos, sexuales y reproductivos, además de tener consciencia respecto al contexto de opresión reproductiva en el que se encuentran las mujeres (Ross y Solinger, 2017).

La justicia reproductiva es un movimiento que parte de mostrar la necesidad de que la defensa de los derechos reproductivos vaya de la mano de la búsqueda de justicia social (Ross y Solinger, 2017); de evidenciar que las mujeres, a pesar de tener el derecho legal a decidir sobre su cuerpo, no pueden ejercerlo si están inscritas en un contexto en el que las elecciones 
en torno a su reproducción no ocurren de manera accesible, autónoma, informada, digna y segura.

Este movimiento fue creado por las feministas negras estadounidenses en respuesta al pasado colectivo de esclavización de sus comunidades ejercida a través del control de la capacidad reproductiva de las mujeres (Ross y Solinger, 2017). La justicia reproductiva busca que, en las políticas sexuales y reproductivas, se tomen en cuenta las necesidades diversas que tienen las mujeres de acuerdo con la interseccionalidad de factores de clase, raza, género, orientación sexual, capacidades, estado de salud y estatus migratorio (Ross y Solinger, 2017).

Al tener en sus pilares de atención el acompañar e informar a las mujeres para que tomen decisiones autónomas, así como el respeto y defensa de sus derechos, el modelo de partería ofrece una atención a la salud que garantiza justicia social. La justicia reproductiva se piensa como un bienestar económico, político, físico, mental y espiritual (Asian Communities for Reproductive Justice, 2005). Las parteras, al ofrecer atención integral que toma en cuenta tanto la atención del cuerpo físico como las emociones y los procesos de espiritualidad y significación que las mujeres tienen en torno a su vida reproductiva, garantizamos el bienestar integral en los ámbitos de la sexualidad y la reproducción.

\section{Modelo de partería}

Las parteras somos profesionales autónomas, expertas en la atención de la salud sexual y reproductiva de las mujeres desde el modelo de partería (en adelante lo designaremos MdP). Muchas parteras brindan atención únicamente durante la gestación, el parto y el postparto; otras no limitan su práctica a la atención perinatal, sino que están capacitadas para atender la mayoría de los procesos sexuales y reproductivos, como la atención pregestacional, el apoyo a la fertilidad, la salud menstrual y vaginal y, en algunos países, la interrupción del embarazo.

El MdP se ubica dentro del primer nivel de atención a la salud; es decir, el área de especialidad de las parteras son los procesos fisiológicos (sanos) de las mujeres, como la gestación y el parto; procesos que, según la OMS (2018), en la mayoría de los casos se pueden llevar a cabo sin complicaciones. Las parteras también estamos capacitadas para realizar intervenciones sencillas, atender algunos desequilibrios en la salud y 
resolver algunas emergencias obstétricas (International Confederation of Midwives [ICM], 2019).

Una piedra angular de la modalidad de atención de las parteras -y que difiere completamente del modelo ginecobstétrico- es el enfoque integral y centrado en cada mujer. La relación partera-mujer ${ }^{2}$ está mediada por un vínculo que se construye a través de la continuidad de atención. El papel de la partera es brindar información basada en evidencia y acompañamiento personalizado que se acople a las necesidades y características específicas de cada mujer sin tomar un papel protagónico en la toma de decisiones y confiando en la capacidad y agencia de las mujeres para elegir de forma libre y autónoma.

Además, las parteras entendemos que la sexualidad, el embarazo, el parto y el aborto son procesos de transformación en la vida de las mujeres con una gran complejidad y una carga emocional profunda, en muchos de los casos.

La práctica de las parteras se basa en la defensa y protección de los derechos de las mujeres, como el derecho a decidir de forma libre e informada sobre su cuerpo y su sexualidad, el acceso a servicios de salud en los que se les brinde una atención digna y respetuosa, el acceso a la información detallada sobre sus procesos de salud y el derecho a no ser discriminadas ni violentadas. Por ello, algunas parteras nos posicionamos como defensoras de los derechos de las mujeres además de como profesionales de la salud.

El MdP tiene beneficios tanto para la salud de las mujeres como para los sistemas de salud. Por un lado, es un modelo de atención seguro, no tiene efectos adversos (Homer, 2016), reduce la incidencia de complicaciones y de intervenciones innecesarias y mejora las experiencias y la satisfacción de las mujeres (Olsen y Clausen, 2012). Por otro lado, es una estrategia para contrarrestar la violencia ginecobstétrica. Finalmente, por la baja incidencia de intervenciones, es una práctica costo-efectiva y, como se lleva a cabo en casa, casas de partería o infraestructura de salud del primer nivel, trasladar

2 No utilizamos la palabra "paciente" (del latín patientis, participio de pati, "padecer"), ya que hace alusión al padecimiento de una enfermedad y nuestra práctica se centra en las mujeres sanas. Además de que tiene una connotación pasiva que le resta agencia al sujeto. En el MdP, las mujeres tienen agencia y protagonismo en todo momento, ya que la toma de decisiones se realiza de manera conjunta. 
la atención de las mujeres sanas o de bajo riesgo a la atención por parteras contribuye a desaturar y equilibrar el sistema hospitalario (Homer, 2016) .

\section{Partería y aborto en el mundo}

De acuerdo con la Organización de las Naciones Unidas (ONU), las parteras podríamos dar el $87 \%$ de la atención esencial a la salud sexual y reproductiva de las mujeres (Iro, 2018) y eso incluye abortos. En países en donde las parteras están reguladas y, en algunos casos, integradas al y respaldadas por el sistema de salud, su rol como proveedoras está claro y descrito, lo cual contribuye a la aceptación de que provean servicios de aborto, como es el caso de Suecia (Sorhaindo, 2020, p. 51). En los países en donde las parteras estamos fuera del sistema gubernamental de salud, como en México, formamos parte de lo que llamaremos servicios alternativos de salud. En esos países, las parteras también llegan a ser acompañantes de abortos, proveedoras de servicios y defensoras del derecho de las mujeres a elegir sobre sus cuerpos, aunque no sean formalmente reconocidas como tales.

La OMS recomienda incluir a parteras como proveedoras de salud esenciales en la oferta de servicios de aborto (World Health Organization [WHO], 2015), tanto para aborto con medicamentos como para aspiración manual endouterina (AMEU). Además, sugiere que podamos dar consejería preaborto, consejería de antifecundativos e iniciar tratamiento para hemorragias e infecciones postaborto. Aunado a lo anterior, la OMS evidencia que la atención de procesos de aborto por parteras es percibida por las mujeres como un servicio de mayor calidez (WHO, 2015). El que no sólo personal médico provea servicios de aborto, sino que se incluyan a otros profesionales de la salud como las parteras, es una propuesta de task-sharing (delegación y repartición de funciones) de la OMS y se plantea como una solución para reducir los casos de abortos inseguros y reducir la brecha existente entre las mujeres que necesitan abortos y el personal de salud capacitado para atenderlas (WHO, 2015).

La misma posición es defendida por la Confederación Internacional de Parteras (ICM por sus siglas en inglés): "las mujeres buscando abortos [...] deben poder recibir ese servicio por parte de parteras" (International Confederation of Midwives [ICM], 2014, p. 1). ${ }^{3}$ En Estados Unidos, por

3 En el original: "ICM affirms that a woman who seeks or requires abortion-related services is entitled to be provided with such services by midwives".

Borboleta, H., Cisneros, K. y Llanes, R. (2022). Modelo de partería, justicia reproductiva y atención de aborto en México. Iberoforum, Revista de Ciencias Sociales, Nueva Época, 2(1), 1-17, Ensayos, e000198. https://doi.org/10.48102/if.2022.v2.n1.198 
ejemplo, el Colegio de Parteras Enfermeras Certificadas (ACNM por sus siglas en inglés) respalda a sus integrantes para que hagan abortos y afirma que en varios estados no tienen que ser médicas o médicos quienes den ese servicio (American College of Nurse Midwives [ACNM], 2018) y que las parteras enfermeras son un recurso necesario y recomendable para cerrar la brecha entre las y los proveedores de aborto que debería haber y las y los que en realidad hay.

En otros países, como Canadá, Reino Unido, Francia y Sudáfrica (Dickson-Tetteh y Billings, 2002), también se presenta a las parteras como proveedoras de salud competentes y necesarias para realizar abortos. Las posibilidades de acción de las parteras dentro de los servicios de aborto varían según el país: en algunos, pueden prescribir mifepristona y misoprostol, pero no hacer AMEU; en otros, pueden hacer todo lo anterior. En lugares donde la partería carece de regulación estatal, pero hay parteras formadas en atención de aborto, a veces usan medicina complementaria para el aborto mismo y apoyan con la situación emocional de las mujeres, el manejo del dolor y la prevención o el tratamiento de complicaciones. Además, es probable que las parteras den atención en sus propias casas o en casa de las mujeres sin necesitar la infraestructura hospitalaria.

\section{Partería mexicana y aborto}

En México, la gran mayoría de parteras atienden en casa. Exponemos aquí una clasificación de los diferentes tipos de partería que existen actualmente en el país: parteras profesionales - aquéllas con título y formadas en escuelas reconocidas por el Estado-, parteras tradicionales o desde la tradición -aquéllas que se forman en un modelo de maestra-aprendiz a través de la transmisión de saberes ancestrales y poniendo en el centro la espiritualidad y la conexión con lo sagrado (Gallegos, 2019) - y parteras autónomas - parteras de diversos caminos de formación que suelen formarse y ejercer fuera del sistema de salud aunque no forzosamente con una formación en la tradición-. Dada la falta de reconocimiento de las parteras por parte del Estado mexicano como personal de salud capacitado para atender la salud materno-infantil, se complica su aceptación y reconocimiento como profesionales capaces de atender abortos (Schiavon y Troncoso, 2020).

A pesar de las recomendaciones internacionales que proponen la delegación de funciones en los servicios de aborto, las legislaciones mexicanas únicamente permiten que personal médico atienda abortos, lo cual 
dificulta el acceso a las mujeres, ya que es mayor la demanda en comparación con la cantidad de personal médico que puede dar ese servicio (Schiavon y Troncoso, 2020). Todas las parteras se pueden formar en el uso de misoprostol y mifepristona y en AMEU con apoyo de escuelas y colectivos, aunque el equipo con el que trabajan corre por su cuenta o es producto de donaciones.

A diferencia del modelo biomédico, los protocolos de atención en el MdP priorizan las necesidades de las mujeres. A continuación, ejemplificaremos el ejercicio de algunas parteras que acompañan abortos. ${ }^{4}$ Sus consultas suelen durar como mínimo una hora; durante ese tiempo, explican las posibilidades de procedimientos, riesgos y beneficios y ponen en el centro la decisión de las mujeres. Las parteras siempre les preguntan a las mujeres si entendieron la información; hacen espacios para preguntas y no realizan ninguna intervención hasta que las mujeres estén listas y hayan dado su consentimiento explícitamente. Este último elemento es clave, ya que las mujeres lo mencionan repetidamente como una de las grandes diferencias que notan en la atención: ellas están en el centro y son las que deciden qué se hace y cómo; no las proveedoras. Según una encuesta informal a un equipo de parteras que acompañan abortos en México, las usuarias mencionan que su experiencia fue amena y el servicio que recibieron fue de calidad por no sentirse juzgadas, por recibir explicaciones sobre el procedimiento, por ser escuchadas y porque no se les pidieron explicaciones respecto a su proceso de interrupción.

Mientras que las instancias gubernamentales en los estados que tienen la causal voluntaria ofrecen aborto con medicamento antes de las nueve semanas y AMEU entre las nueve y hasta las doce semanas, las parteras acompañamos las decisiones de las mujeres y defendemos que cada mujer sabe lo que es mejor para ella, sus circunstancias y su estilo de vida. Por ello, la capacitación en servicios de aborto a todas las parteras y su reconocimiento permitiría que demos el servicio que cada mujer elija.

Las parteras también estamos convencidas de que, así como en el parto la supervivencia no es suficiente, en el aborto tampoco: la atención

4 La información fue extraída de la correspondencia personal con parteras que piden mantenerse en el anonimato por la compleja situación legal. 
tiene que ir más allá y asegurar que las mujeres puedan atravesar esos eventos con dignidad, calidad y calidez y ser las protagonistas siempre.

Sabemos, a partir de la experiencia de las redes de acompañantes de aborto en México, que las mujeres tienen experiencias más positivas cuando reciben acompañamiento continuo, presencia y atención sin juicio. Además, tener una relación personalizada con quien te acompaña es importante para la seguridad y la confianza en un proceso de salud (Dahlberg y Aune, 2013) y un aborto no es la excepción.

Es importante recordar que, para que el MdP funcione, las parteras debemos ser profesionales autónomas, no bajo los protocolos de un hospital o del Estado, sino siendo servidoras y acompañantes de las mujeres.

\section{Conclusiones}

La saturación del sistema hospitalario, la escasez de profesionales de salud capacitados para la atención del aborto y la violencia ginecobstétrica sistémica e institucionalizada son realidades que impactan de manera negativa en la salud de las mujeres e impiden el acceso a servicios verdaderamente seguros y dignos de atención de aborto para todas las mujeres.

Las parteras somos profesionales de la salud con las competencias y habilidades necesarias para atender abortos de forma segura. El MdP fomenta la justicia reproductiva, ofrece una atención a la salud que garantiza justicia social, responde a las necesidades específicas de cada mujer, se centra en los derechos humanos y promueve la autonomía de las mujeres.

El reconocimiento de las parteras como profesionales de salud autónomas y expertas en la atención a la salud sexual y reproductiva de las mujeres, así como invertir en la capacitación de parteras para ser la primera línea de atención en los servicios de aborto en México, es una estrategia costo-efectiva que contribuiría a garantizar el acceso a una atención segura, digna y respetuosa; mejoraría la experiencia de las mujeres, reduciría las intervenciones innecesarias, disminuiría la violencia ginecobstétrica y desaturaría el sistema hospitalario.

\section{Referencias bibliográficas}

American College of Nurse Midwives (2018). Position statement. Midwives as abortion providers. http://www.midwife.org/acnm/files/acnmlibrarydata/uploadfilename/000000000314/ps-midwives-as-abortion-providers-final-19-mar-18.pdf 
Arguedas, R. G. (2014). La violencia obstétrica: Propuesta conceptual a partir de la experiencia costarricense. Cuadernos Inter.c.a.mbio sobre Centroamérica y el Caribe, 11(1), 145-169. https://www.redalyc.org/ articulo.oa?id=476947241008

Asian Communities for Reproductive Justice (2005). A new vision for advancing our movement for reproductive health, reproductive rights and reproductive justice. Forward Together. https://forwardtogether. org/tools/a-new-vision

Castro, R. y Erviti, J. (2015). Sociología de la práctica médica autoritaria: Violencia obstétrica, anticoncepción inducida y derechos reproductivos. Universidad Nacional Autónoma de México.

Dahlberg, U. y Aune, I. (2013). The woman's birth experience-The effect of interpersonal relationships and continuity of care. Midwifery, 29(4) 409-415. https://doi.org/10.1016/j.midw.2012.09.006

Dickson-Tetteh, K. y Billings, D. (2002). Abortion care services provided by registered midwives in South Africa. International Perspectives on Sexual and Reproductive Health, 28(3), 144-150. https://www.guttmacher.org/journals/ipsrh/2002/09/abortion-care-services-provided-registered-midwives-south-africa

Ehrenreich, B. y English, D. (1990). Por tu propio bien: 150 años de consejos expertos a las mujeres. Capitán Swing Libros.

Enciso Rojas, D. (2010). "Mal parir", "parir fuera de tiempo" o "aborto procurado y efectuado". Su penalización en Nueva España y el México independiente. Dimensión Antropológica, (49), 91-123. http://www. dimensionantropologica.inah.gob. $\mathrm{mx} / \mathrm{p} \mathrm{p}=4853$

Federici, S. (2004). Calibán y la bruja. Mujeres, cuerpo y acumulación primitiva. Traficantes de Sueños.

Gallegos, A. R. (2019). Formar parteras desde la tradición: Estrategia para la continuidad de la partería en Oaxaca y México [Documento recepcional]. Universidad Veracruzana. https://www.uv.mx/meis/ files/2020/01/DR-Aura-Gallegos-MEIS-1.pdf

Grupo de Información en Reproducción Elegida (2018). Maternidad o castigo. La criminalización del aborto en México. GIRE. https://criminalizacionporaborto.gire.org.mx/assets/pdf/Maternidad_o_castigo.pdf

Homer, C. S. (2016). Models of maternity care: Evidence for midwifery continuity of care. The Medical Journal of Australia, 205(8), 370-374. https://doi.org/10.5694/mja16.00844 
Iro, E. (2018). Midwives are essential to the provision of quality of care, in all settings, globally. https://www.who.int/news-room/commentaries/ detail/midwives-are-essential-to-the-provision-of-quality-of-care-in-all-settings-globally

International Confederation of Midwives (2019, octubre). Essential Competencies for Midwifery Practice. https://internationalmidwives.org/assets/files/general-files/2019/10/icm-competencies-en-print-october-2019_final_18-oct-5db05248843e8.pdf

International Confederation of Midwives (2014). Position statement. Midwives provision of abortion-related services. https://www.internationalmidwives.org/assets/files/statement-files/2018/04/ midwives-provision-of-abortion-related-services-eng.pdf

Núñez, F. (2008). Imaginario médico y práctica jurídica en torno al aborto durante el último tercio del siglo XIX. En C. Agostini (ed.), Curar, sanar y educar. Enfermedad y sociedad en México, siglos XIX y XX. Universidad Nacional Autónoma de México. https://www.historicas.unam.mx/publicaciones/publicadigital/libros/curar_ sanar/494_04_05_imaginario.pdf

Olsen, O. y Clausen, J. A. (2012). Planned hospital birth versus planned home birth. Cochrane Database of Systematic Reviews, 9. https://doi. org/10.1002/14651858.CD000352.pub2

Organización Mundial de la Salud (2020). Prevención del aborto peligroso. https://www.who.int/es/news-room/fact-sheets/detail/preventing-unsafe-abortion

Organización Mundial de la Salud (2018). Recomendaciones de la OMS. Para los cuidados durante el parto, para una experiencia de parto positiva. Transformar la atención a mujeres y neonatos para mejorar su salud y bienestar. Resumen de orientación. https://apps.who.int/iris/ handle $/ 10665 / 272435$

Palomar, V. C. (2004). Malas madres: La construcción social de la maternidad. Debate Feminista, 30, 12-34. https://doi.org/10.22201/ cieg.2594066xe.2004.30.1046

Ross, L. y Solinger, R. (2017). Reproductive Justice, an Introduction. University of California Press.

Schiavon, R. y Troncoso, E. (2020). Inequalities in access to and quality of abortion services in Mexico: Can task-sharing be an opportunity to increase legal and safe abortion care? International Journal Gyneco- 
logy Obstetrics, 150(S1), 25-33. https://doi.org/10.1002/ijgo.13002

Secretaría de Salud (2021). Interrupción legal del embarazo (ILE). Estadísticas. Abril 2007-30 de septiembre 2021. http://ile.salud.cdmx.gob. $\mathrm{mx}$ /estadisticas-interrupcion-legal-embarazo-df/

Singh, S., Remez, L., Sedgh, G., Kwok, L. y Tsuyoshi, O. (2018). Abortion Worldwide 2017: Uneven Progress and Unequal Access. Guttmacher Institute.https://www.guttmacher.org/sites/default/files/report_pdf/ abortion-worldwide-2017.pdf

Sorhaindo, A. (2020). Creativity, serendipity, and collaboration: Crosscutting features of successful task-sharing in comprehensive safe abortion care. International Journal of Gynecology and Obstetrics, 150(S1), 49-54. https://doi.org/10.1002/ijgo.13011

Stern, A. (2002). Madres conscientes y niños normales: La eugenesia y el nacionalismo en el México posrevolucionario, 1920-1940. En L. Cházaro (ed.), Medicina, ciencia y sociedad en México, siglo XIX (pp. 293-336). El Colegio de Michoacán, Universidad Michoacana de San Nicolás de Hidalgo.

World Health Organization (2015). Health Workers Role in Providing Safe Abortion Care and Post-abortion Contraception. http://apps.who. int/iris/bitstream/handle/10665/181041/9789241549264_eng.pdf 